\title{
Pseudomonas-Contaminated Pool Triggering an Episode of Idiopathic Palmoplantar Hidradenitis
}

\author{
Rasheed K. Barradah \\ Department of Dermatology, College of Medicine, Majmaah University, Almajmaah, Saudi \\ Arabia
}

\section{Keywords}

Hot-foot syndrome · Pseudomonas · Idiopathic palmoplantar hidradenitis · Idiopathic recurrent palmoplantar hidradenitis - Palmoplantar eccrine hidradenitis

\begin{abstract}
Idiopathic palmoplantar hidradenitis (IPPH) is a disorder that mainly affects the palms and soles of children. Although many cases have been reported to occur after recreational swimming activities, whether IPPH is caused by intense physical activity or by Pseudomonas infection has yet to be proven. We report a case of a 3-year-old girl who presented with IPPH after swimming in a pool with evidence of $P$. aeruginosa contamination, further solidifying the association between Pseudomonas and IPPH.
\end{abstract}

\section{Introduction}

The term "palmoplantar eccrine hidradenitis" was first coined in 1998 although the disorder was described as early as 1994 in children that presented with tender and erythematous plantar nodules $[1,2]$. Pseudomonas hot-foot syndrome (HFS), which was first described in 2001, is a disease caused by infection with $P$. aeruginosa and presents with clinical features identical to palmoplantar eccrine hidradenitis [3]. The term "hot-foot syndrome" has also been reported as "a combination of a hot foot, ankle edema, and pain associated with recurrent retroperitoneal tumors" - this is a completely different entity and is beyond our scope of discussion [4]. Whether HFS is a distinct entity or not from idiopathic palmoplantar hidradenitis (IPPH) remains a debate. However, establishing an association between IPPH and $P$. aeruginosa will aid in improving prevention and treatment efforts while highlighting the 
disease as an important public health concern especially for children who frequent public swimming pools [5].

\section{Case Report}

A 3-year-old previously healthy girl presented with difficulty in walking 1 day after swimming for $1 \mathrm{~h}$ in a backyard pool during the summer. The pool was filled 1 week prior with unchlorinated water drawn from a nearby artesian well. The patient reportedly had a low-grade fever, could barely walk on her heels, and frequently laid down crying from pain. On examination, she had ill-defined erythematous nodules on her forefoot and on the digital pulp of the toes on both feet (shown in Fig. 1a, b). No other skin lesions were noted. Her father reported that her 2 siblings also experienced similar but less severe symptoms. Her past medical history was unremarkable. The patient's mother soaked her feet in salt and lime, applied petroleum jelly, and gave her bed rest, all of which reportedly decreased the pain. All lesions resolved within 3 days with minimal peeling of the skin. A water sample was taken for testing from the residual water on the smooth plastic flooring of the pool. The results of the test are as follows: $\mathrm{HPC}>300 \mathrm{CFU} / \mathrm{mL}$, total coliforms $=28 \mathrm{CFU} / 100 \mathrm{~mL}$, fecal coliforms $<1$ $\mathrm{CFU} / 100 \mathrm{~mL}$, and $P$. aeruginosa $=86 \mathrm{CFU} / 100 \mathrm{~mL}$.

\section{Discussion}

\section{Clinical Presentation}

IPPH presents with an abrupt onset of erythematous, tender nodules on the plantar area leading to discomfort when walking; however, IPPH occasionally presents with pustules and may affect the palms. Systemic symptoms are usually absent but rare cases that presented with a low-grade fever have been reported $[3,6]$. The presence of necrotic nodules and systemic symptoms should prompt investigation for an underlying immunosuppression [7].

\section{Pathogenesis}

The pathogenesis of IPPH remains unknown. However, previous studies have presented several hypotheses including local thermal or mechanical trauma; pedal perspiration; moisture; intense physical activity; vibration; recent infections, especially streptococcal ones; recent vaccination; hypersensitivity reactions; and Pseudomonas infection of mechanically stressed areas of the skin, especially the soles, which could provide a portal of entry to the eccrine glands [2, 3, 6, 8-12]. Some authors have reported a higher incidence of IPPH during the spring and summer seasons $[10,11]$, leading to the hypothesis that the eccrine glands of pediatric patients may be functionally immature and therefore easily damaged by increases in temperature [10], mechanical trauma, and friction. These insults ultimately lead to the rupture of eccrine glands and the subsequent release of sweat to adjacent tissues, thereby activating inflammation and subsequent neutrophil recruitment $[10,13]$. The association between IPPH and pools infected with Pseudomonas is supported by the following factors: patients develop lesions less than a week after swimming in pools [3, 5, 7], outbreaks have been reported in patients who used the same pool $[3,14,15]$, skin lesion culture-positive for Pseudomonas in a few cases $[7,14]$, lesions recurred in patients who repeatedly visited pools $[3,16]$, disease incidence decreased after disinfection of pool floors but not after reducing floor roughness, and genetic analysis of Pseudomonas isolates from patients with HFS and pool waters revealed a single strain [3]. One explanation of why children develop IPPH more

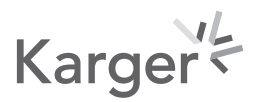




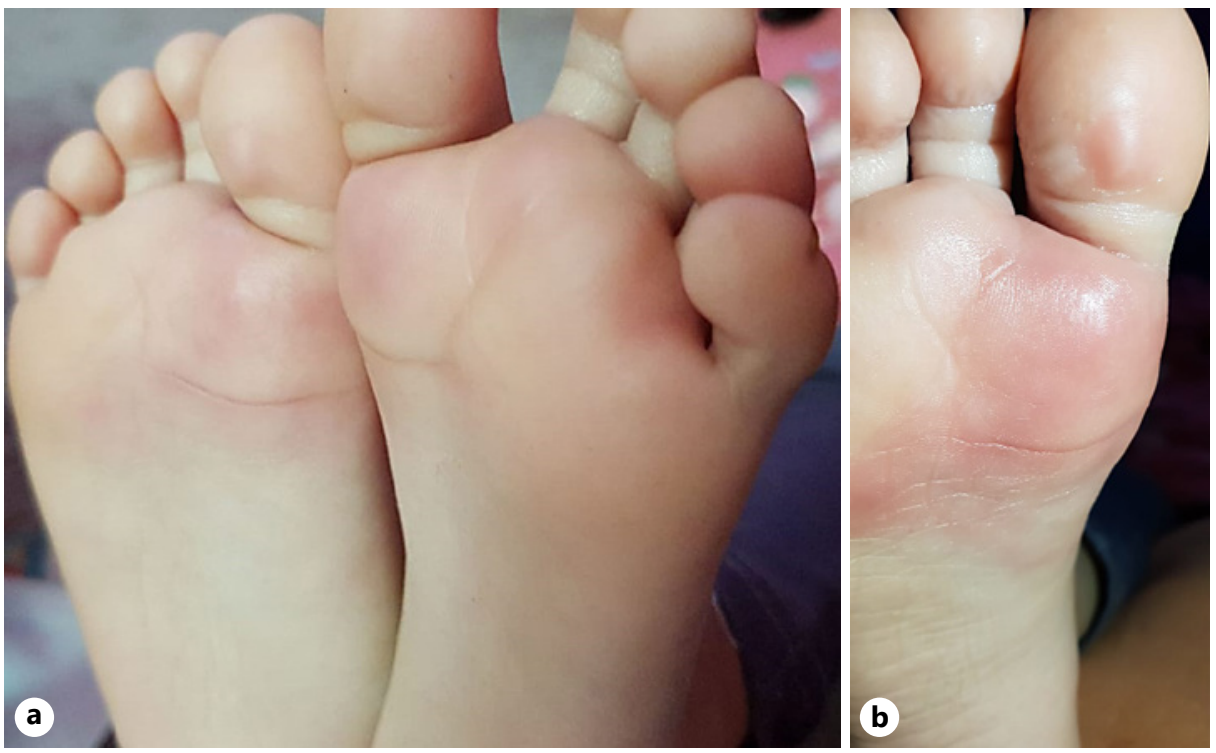

Fig. 1. a, b Bilateral ill-defined erythematous tender nodules over the forefoot.

often than adults is that the epidermis on their palms and soles are thinner [5]. Furthermore, children have increased contact with the floor due to their high mobility [17] and their tendency to "swim" by standing in shallow waters. It is hypothesized that Filaggrin-2 (FLG2), a water-insoluble surface antimicrobial protein present in eccrine glands and in the stratum corneum, directly inhibits the growth of $P$. aeruginosa [18]. This protein is known to be absent on certain body surfaces such as the lungs and burned skin [18]. We hypothesize that exposure to friction burn and subsequent infection of the palms and soles of children may decrease FLG2 levels and increase the risk for Pseudomonas HFS.

\section{Differential Diagnoses}

Several skin diseases present with palmoplantar involvement; some are associated with exposure to infected water (i.e., hot tub folliculitis), Mycobacterium abscessus infection, or underlying disease (i.e., neutrophilic eccrine hidradenitis), while others are iatrogenic in origin such as in chemotherapy-induced hand-foot syndrome. Other differential diagnoses include hand-foot disease from enteroviruses, erythema multiforme, nodular erythema, plantar erythema nodosum, vasculitis, cold panniculitis, and cellulitis $[9,19]$. Hot tub folliculitis is another skin disease associated with P. aeruginosa infection and presents with pustules and papules on areas of the skin that came in contact with infected water; this disease is also known to occasionally present with nodules exclusively on the palms and soles [3, 5]. Hand-and-foot disease from M. abscessus infection is also acquired from exposure to contaminated water and presents with painful nodules and abscesses. This disease has a long incubation period (median of 58 days) and takes a longer period to resolve [20]. Neutrophilic eccrine hidradenitis is found mainly in patients with leukemia and other neoplastic diseases but can also arise secondary to chemotherapy drugs [21]. Affected locations are usually random and include the trunk, face, and extremities; most lesions present as tender erythematous papules and have extra histopathological features that differentiate them from IPPH $[1,12,22]$. Hand-foot disease from enteroviruses usually presents with asymptomatic vesicular lesions on the hands, feet, and oral cavity [3]. Chemotherapy-induced hand-foot syndrome is commonly associated with sharply demarcated areas of erythema rather than nodules [23]. 


\section{Investigations}

Skin biopsy is the gold standard for diagnosing IPPH. However, considering the typical clinical presentation of IPPH, a biopsy is often not necessary for diagnosis [3,9] except in cases with an abnormally prolonged duration or an atypical presentation [24]. Histopathologic examination will reveal a primarily neutrophilic inflammatory infiltrate surrounding eccrine sweat glands $[8,25]$. Neutrophilic eccrine hidradenitis can be differentiated from IPPH by the presence of necrotic eccrine glands, syringosquamous metaplasia, and neutrophilic infiltrates affecting the ductal and secretory components of the eccrine coils with or without eosinophilic deposits in the basement membrane of the eccrine duct [1, 12, 21, 22]. Histopathologic examination of IPPH will reveal inflammatory infiltrates surrounding the ductal component of eccrine coils and, frequently, neutrophilic abscesses adjacent to eccrine coils with or without septal panniculitis $[1,12,21,22]$. Compared to IPPH, the inflammatory infiltrate of Pseudomonas HFS exhibits a deeper extension [15]. Culture results from skin lesions are usually negative $[1,25]$. Culturing samples from the floor, and not from the suspected water itself, often reveals significant quantities of $P$. aeruginosa [3]. It should be noted that severe presentations of IPPH should prompt a search for underlying immunosuppression [7].

\section{Treatment}

Making an accurate diagnosis is important to avoid unnecessary treatment since IPPH has a benign course [9]. Management should initially focus on bed rest to decrease sweat secretion and subsequent inflammation [8]. Almost all reported cases of IPPH resolved completely within 4 weeks without any treatment $[8,9]$. When Pseudomonas infection is suspected, treatment may consist of topical antibiotics or systemic antipseudomonal antibiotics if the patient presents with systemic symptoms [3]. Recurrence is reported in approximately $50 \%$ of patients; 1 study reported the successful use of colchicine in treating and preventing recurrent episodes [12]. Another study reported that a short course of low-dose oral corticosteroids failed to reduce the duration of symptoms or prevent the recurrence of IPPH and thus is not recommended $[12,21]$.

\section{Prevention}

Since the exact pathogenesis of IPPH has not been established, measures focused on disease prevention are vital. Such measures include chlorinating pools, using nonabrasive materials for pool floors [5], wearing comfortable and well-fitting shoes during exercise, and keeping the soles dry. According to the Centers for Disease Control and Prevention, the recommended chlorine concentration ranges from 3 to $6 \mathrm{ppm}$ and $\mathrm{pH} 7.6-7.8$ for spas and swimming pools, with higher concentrations needed in hot climates [5]. Washing skin exposed to Pseudomonas-infected water does not seem to prevent IPPH [5]. Proper chlorination measures and the use of nonabrasive materials must be combined with complete disinfection of the contaminated floors to effectively prevent Pseudomonas HFS [3].

\section{Conclusion}

Whether Pseudomonas HFS is a distinct entity from IPPH or not remains a debate. We hypothesize that exposure to friction burn and subsequent infection of the palms and soles of children might decrease the quantity of natural defense peptides (i.e., FLG2) and increase the risk for Pseudomonas HFS; this potentially identifies the idiopathic aspect of IPPH. However, further studies are required to prove this hypothesis.

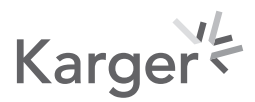




\section{Statement of Ethics}

Written informed consent to publish this case (including images) was obtained from the parents of the patient. The research was conducted ethically in accordance with the World Medical Association Declaration of Helsinki.

\section{Conflict of Interest Statement}

The authors have no conflicts of interest to declare. The author alone is responsible for the content and writing of the paper.

\section{Author Contributions}

Rasheed Khalid Barradah: conception and design, interpretation of data, drafting, and final approval.

\section{References}

1 Stahr BJ, Cooper PH, Caputo RV. Idiopathic plantar hidradenitis: a neutrophilic eccrine hidradenitis occurring primarily in children. J Cutan Pathol. 1994;21(4):289-96.

2 Landau M, Metzker A, Gat A, Ben-Amitai D, Brenner S. Palmoplantar eccrine hidradenitis: three new cases and review. Pediatr Dermatol. 1998;15(2):97-102.

3 Michl RK, Rusche T, Grimm S, Limpert E, Beck JF, Dost A. Outbreak of hot-foot syndrome - caused by Pseudomonas aeruginosa. Klin Padiatr. 2012;224(04):252-5.

4 Paterson IC. Hot foot syndrome. Br Med J. 1978;1:787.

5 Yu Y, Cheng AS, Wang L, Dunne WM, Bayliss SJ. Hot tub folliculitis or hot hand-foot syndrome caused by Pseudomonas aeruginosa. J Am Acad Dermatol. 2007;57(4):596-600.

6 Brites MM, Tellechea O, Baptista AP. Plantar hidradenitis. Acta Medica Port. 2001;14(3):371-4.

7 Laffitte E, Hohl D, Panizzon RG. Pseudomonas eccrine hidradenitis in a child revealing acute lymphoblastic leukemia. Ann Dermatol Venereol. 2004;131(11):975-8.

8 Rubinson R, Larralde M, Santos-Muñoz A, Parra V, de Parra NP. Palmoplantar eccrine hidradenitis: seven new cases. Pediatr Dermatol. 2004;21(4):466-8.

9 Piccini P, Venturini E, Bianchi L, Galli L, de Martino M, Bassi A. Bilateral painful erythematous nodules on the sole. J Pediatr. 2018;199:278.

10 Lee GL, Chen AY. Neutrophilic dermatoses: kids are not just little people. Clin Dermatol. 2017;35(6):541-54.

11 Tlougan BE, Podjasek JO, Dickman PS, Hansen RC. Painful plantar papules and nodules in a child. Palmoplantar eccrine hidradenitis (PEH). Pediatr Ann. 2008;37(2):83-7, 87.

12 Scheer HS, Kamarashev J, Weibel L. Successful treatment of recurrent idiopathic plantar eccrine hidradenitis with colchicine. Arch Dermatol. 2012;148(12):1357-9.

13 Weigl L, Eberlein-König B, Ring J, Abeck D. Is recurrent plantar hidradenitis in children induced by exposure to a wet and cold milieu? Br J Dermatol. 2000;142(5):1048-50.

14 Fiorillo L, Zucker M, Sawyer D, Lin AN. The pseudomonas hot-foot syndrome. N Engl J Med. 2001;345(5): 335-8.

15 Zvulunov A, Trattner A, Naimer S. Pseudomonas hot-foot syndrome. N Engl J Med. 2001;345(22):1643-4.

16 Cremer H. Die rekurrierende palmoplantare Hidradenitis im Kindesalter. Pädiatrie Hautnah (Deutsche Gesellschaft für interdisziplinäre pädiatrische Dermatologie (DGipD). 20031997 4:192-3.

17 Handrick W, Nenoff P. Hot-foot-syndrom oder palmoplantare Hidradenitis? Akt Dermatol. 2006;32(08/09): 357-9.

18 Hansmann B, Schröder JM, Gerstel U. Skin-derived C-terminal filaggrin-2 fragments are Pseudomonas Aeruginosa-directed antimicrobials targeting bacterial replication. PLoS Pathog. 2015;11(9):e1005159.

19 Sandraps E, Blomme S, Demeester A, Decroix J, Marot L, Lachapelle JM. Painful nodular and plantar erythema in children. Ann Dermatol Venereol. 1996;123(10):647-50.

20 Dytoc MT, Honish L, Shandro C, Ting PT, Chui L, Fiorillo L, et al. Clinical, microbiological, and epidemiological findings of an outbreak of Mycobacterium abscessus hand-and-foot disease. Diagn Microbiol Infect Dis. 2005; 53(1):39-45. 
21 Drake M, Sánchez-Bursón JM, Doña-Naranjo MA, Conde JM. Juvenile neutrophilic eccrine hidradenitis: a vasculitis-like plantar dermatosis. Clin Rheumatol. 2000;19(6):481-3.

22 Andreu-Barasoain M, Calzado L, Salamanca J, Nuño A, Gamo R, Gomez de la Fuente E, et al. Generalized idiopathic neutrophilic eccrine hidradenitis in a 7-month-old child. J Am Acad Dermatol. 2012;67(4):e133-4.

23 Qiao J, Fang H. Hand-foot syndrome related to chemotherapy. CMAJ. 2012;184:E818.

24 Erro-Vincent T, Souillet AL, Fouilhoux A, Kanitakis J, David L. Eccrine neutrophilic hidradenitis: idiopathic plantar form in children. Arch Pediatr. 2001;8:290-3.

25 Bartolo E, Anes I, Capitao-Mor M, Brandao FM. Idiopathic plantar hidradenitis. J Eur Acad Dermatol. 1998;10: 257-61. 